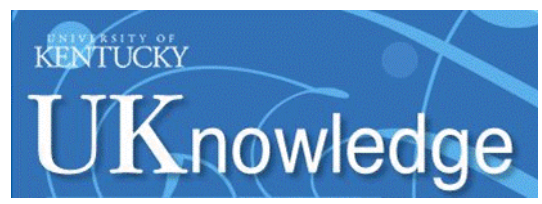

University of Kentucky

UKnowledge

3-21-2016

\title{
Resident Training Curriculum in Adolescent Depression and Suicide Screening
}

\author{
Angela Stanley \\ Medical College of Wisconsin \\ Gayathri Chelvakumar \\ The Ohio State University \\ Paula Cody \\ University of Wisconsin - Madison \\ Mandakini Sadhir \\ University of Kentucky, m.sadhir@uky.edu \\ Melodee Nugent \\ Medical College of Wisconsin
}

See next page for additional authors

Follow this and additional works at: https://uknowledge.uky.edu/pediatrics_facpub

Part of the Curriculum and Instruction Commons, Medical Education Commons, Mental and Social Health Commons, and the Pediatrics Commons

Right click to open a feedback form in a new tab to let us know how this document benefits you.

\section{Repository Citation}

Stanley, Angela; Chelvakumar, Gayathri; Cody, Paula; Sadhir, Mandakini; Nugent, Melodee; Hoffmann, Raymond; and Simpson, Pippa, "Resident Training Curriculum in Adolescent Depression and Suicide Screening" (2016). Pediatrics Faculty Publications. 271.

https://uknowledge.uky.edu/pediatrics_facpub/271

This Article is brought to you for free and open access by the Pediatrics at UKnowledge. It has been accepted for inclusion in Pediatrics Faculty Publications by an authorized administrator of UKnowledge. For more information, please contact UKnowledge@lsv.uky.edu. 
Resident Training Curriculum in Adolescent Depression and Suicide Screening

Digital Object Identifier (DOI)

https://doi.org/10.15766/mep_2374-8265.10361

Notes/Citation Information

Published in MedEdPORTAL, v. 12, 10361, p. 1-5.

(C) 2016 Stanley et al.

This is an open-access publication distributed under the terms of the Creative Commons AttributionNonCommercial-Share Alike license.

Authors

Angela Stanley, Gayathri Chelvakumar, Paula Cody, Mandakini Sadhir, Melodee Nugent, Raymond Hoffmann, and Pippa Simpson 


\section{Resident Training Curriculum in Adolescent Depression and Suicide Screening}

Angela Stanley, PsyD*, Gayathri Chelvakumar, MD, Paula Cody, MD, Mandakini Sadhir, MD, Melodee Nugent, Raymond Hoffmann, PhD, Pippa Simpson, PhD

*Corresponding author: astanley@mcw.edu

\section{Abstract}

Introduction: Routine screening of adolescents for depression and suicide risk is now considered best clinical practice. However, due to a lack of training, many physicians do not engage in screening. The goal of this curriculum is to improve learner knowledge of and comfort in adolescent depression and suicide risk assessment and management. Methods: The didactic session typically takes 60-90 minutes to complete. In addition to a teaching PowerPoint that provides instruction in both the assessment and management of adolescent depression and suicide risk, the curriculum also includes a suicide assessment and management protocol. This protocol outlines an algorithm that assists in distinguishing between individuals at high and low risk of suicide. The algorithm enables clinicians to determine the most appropriate management plan based on the assessed level of suicide risk. Results: Outcome measures indicate that the majority of residents improved their self-perceived knowledge and comfort in assessing and managing depression and suicide risk, with an average of $61.7 \%$ of residents moving from the novice to the proficient group at the conclusion of the rotation. These results reflect learners obtaining 4 weeks of supervised clinical experience after the didactic session. Discussion: This curriculum was designed for use with pediatric and internal medicine-pediatric residents during their adolescent medicine rotation but could easily be adapted for use with other learners in different settings.

\section{Keywords}

Depression, Adolescent, Suicide, Mental Health, Educational Curriculum

\section{Educational Objectives}

By the end of this module, the learner will be able to:

1. Recognize current professional guidelines and best practice recommendations for adolescent depression and suicide screening.

2. Describe the prevalence and epidemiology of adolescent depression and suicide.

3. Summarize risk and protective factors for adolescent depression and suicide.

4. Identify essential components of a thorough suicide risk assessment and accurately determine level of suicide risk.

5. State specific steps in effective management of suicidal patients corresponding with assessed level of risk.

6. Summarize outcome data regarding effectiveness of the training curriculum.

\section{Introduction}

With the lifetime prevalence of a depressive disorder for adolescents through age 18 estimated to be as high as $20 \%^{1}$ and suicide being the second leading cause of death in the United States for individuals aged $15-24,{ }^{2}$ screening for these conditions has become a highly recommended best clinical practice. ${ }^{3-5}$ In 2009 the US Preventive Services Task Force issued a recommendation that adolescents aged 12-18 seen

Citation: Stanley A, Chelvakumar G, Cody $\mathrm{P}$, et al. Resident training curriculum in adolescent depression and suicide screening. MedEdPORTAL. 2016;12:10361. https://doi.org/10.15766/ mep_2374-8265.10361

Copyright: $\odot 2016$ Stanley et al. This is an open-access publication distributed under the terms of the Creative Commons Attribution-NonCommercialShare Alike license.

\section{Appendices}

A. Resident Training Session .pptx

B. Suicide Assessment Management Protocol.docx

C. Safety Plan Worksheet docx

D. Patient Information Letter docx

All appendices are peer reviewed as integral parts of the Original Publication. 
in primary care medical settings complete a universal screening for major depressive disorder. ${ }^{6}$ The American Academy of Pediatrics recently extended the recommended screening ages to $11-21 .{ }^{4}$

Despite the pervasiveness of adolescent depression and suicide and the strong recommendation to screen for these conditions, many physicians do not engage in screening due to a self-reported lack of confidence, skills, and knowledge, ${ }^{7}$ as well as lack of orientation to psychosocial aspects of medical care. ${ }^{8}$ In a systematic review, Zuckerbrot and Jensen concluded that most physicians do not systematically screen for depression and that the recognition of adolescent depression in primary care would greatly improve with physician training and consistent use of depression rating scales. ${ }^{9} \mathrm{~A}$ recent survey of pediatric residency program directors indicated a general lack of focus on mental health training in pediatric residency programs, resulting in resident knowledge and skill deficits ${ }^{10}$ and, ultimately, substandard patient care.

This curriculum was created in response to these empirically identified needs. The curriculum has been taught for the past 3 years to pediatric and internal medicine-pediatric residents during their adolescent medicine rotation at a large midwestern medical school. It consists of a 60- to 90-minute didactic session followed by supervised clinical practice during the 4-week rotation. The overall goal of the curriculum is to improve learner knowledge and comfort in adolescent depression and suicide assessment and management.

This program builds upon prior work in the area, including current MedEdPORTAL publications of webbased $^{11}$ and team-based ${ }^{12}$ learning suicide risk assessment modules, by extending depression and suicide-screening training from a single intervention to an integrated curriculum. The curriculum is unique in that it provides learners with preceptorship and in vivo, supervised practice. The suicide assessment and management protocol (SAMP; Appendix B) and safety plan (Appendix C) are unique, user-friendly, time-efficient tools that assist in effectively determining individuals at acute risk of suicide and applying interventions appropriate to the identified level of risk. These tools, which were specifically created for this curriculum, have been well-received by both residents and faculty and have been integrated with the electronic medical record at the Medical College of Wisconsin for general use throughout the health system.

This resource meets an identified need for an effective curriculum to improve the ability of pediatric residency programs to train pediatricians in integrating mental health care into routine clinical practice, consistent with current guidelines. ${ }^{3-6,10}$ Providing training during residency will likely have a long-term impact on pediatricians' mental health screening and management practices, fostering enhanced recognition and effective, timely interventions for adolescents with depression and heightened suicide risk. Widespread use of this curriculum in pediatric residency programs has great potential for improving adolescent mental health on a population-wide basis.

\section{Methods}

In addition to a teaching PowerPoint providing instruction in both the assessment and management of adolescent depression and suicide risk, the curriculum also includes an SAMP algorithm that enables clinicians to quickly determine the most appropriate management plan based on the patient's assessed level of suicide risk. The SAMP also includes a safety plan worksheet. The curriculum goes beyond simply teaching individuals how to administer and score a screening measure and helps to ensure that learners are competent in effectively managing the information obtained during the assessment.

The depression screener used in this curriculum is the Patient Health Questionnaire-9 (PHQ-9), Modified for Teens version. The PHQ-9 Modified for Teens has been found to have good sensitivity, specificity, and construct validity in health care settings with an adolescent population, ${ }^{11}$ is easy to administer and score, and is freely and publicly available on the Internet at http://www.cappcny.org/home/documents/PHQ9\%20modified\%20teens.pdf. The PHQ-9 Modified for Teens contains the same items as the original PHQ9 , with minor wording changes, and three additional qualitative questions that are not included in the scoring of the instrument. 
This curriculum was designed for use with pediatric and internal medicine-pediatric residents during their adolescent medicine rotation. However, the curriculum could easily be adapted for other learners including residents from other disciplines, fellows, primary care and subspecialty attending physicians, and other professionals who would benefit from integrating adolescent depression and suicide screening into their practice.

In order to implement this curriculum, a thorough review of the PowerPoint Didactic (Appendix A) and SAMP is recommended prior to implementing the curriculum to ensure instructor mastery of the material. Specific information pertaining to resources in the PowerPoint, SAMP, and safety plan worksheet should be modified to reflect available local resources in the users' area prior to using the curriculum. These areas are indicated by bracketed, blue-colored text throughout the materials.

The didactic session typically takes between 60 to 90 minutes to complete. Learners should be given handouts of the PowerPoint didactic, a copy of the PHQ-9 Modified for Teens, the SAMP, the safety plan worksheet, and the patient information letter (Appendix D) prior to the session. The session facilitator should familiarize him or herself with the didactic and be well-versed in screening and managing adolescents with depression and suicidal ideation prior to initiating training.

For the curriculum to be most effective, it is recommended that learners have the opportunity to practice these skills in a clinical setting that has already adopted routine depression and suicide screening, and where experienced supervisors and/or consultants are available.

\section{Results}

The results reflect learners obtaining 4 weeks of supervised clinical experience after the didactic session. Outcome measures indicate that the majority of residents improved their knowledge and comfort in assessing and managing adolescent depression and suicide risk, with an average of $61.7 \%$ of residents moving from the novice to the proficient group at the conclusion of the rotation (see the Figure).
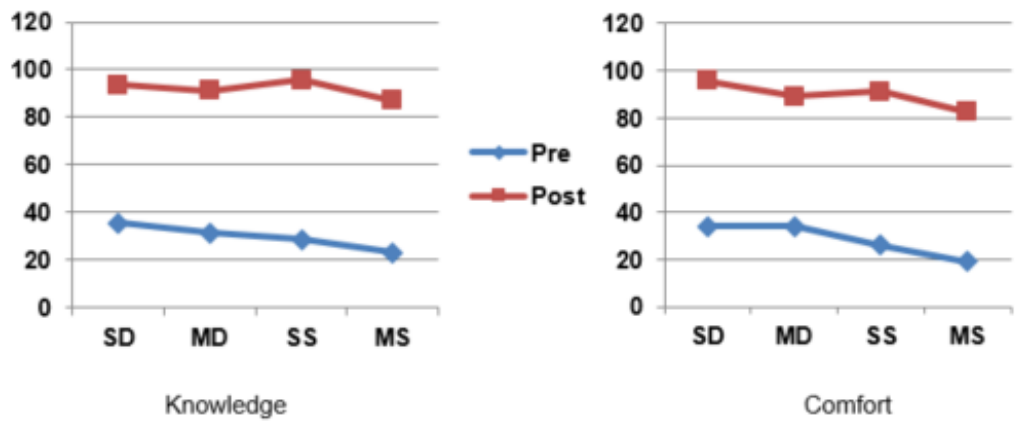

Figure. Percentage of residents who self-rated as Intermediate or Proficient on pretest $(n=73)$ and posttest $(n=46)$. For all comparisons, $p \leq .001$. SD, Screen for Depression; MD, Manage Depression; SS, Screen for Suicidal Ideation; MS, Manage Suicidal Ideation.

\section{Discussion}

In implementing the curriculum, we initially found it helpful to use trial and error in determining the best flow of material and specific content. The didactic was updated to reflect changes in our process and current statistics and guidelines; however, the basic content and flow have remained the same. Users are encouraged to use a similar process to adapt the curriculum to their own needs to ensure continuity in learner outcomes.

Training sessions were conducted in small groups of two to three residents during their adolescent medicine rotation. While teaching in small groups was time consuming, it encouraged active learner engagement and a rich discourse between residents and facilitator. The curriculum could be adapted for use with a classroom or larger group of residents, but this richness would likely be lost. A larger group format would also have an unknown impact on the training's effectiveness. If the curriculum format is 
significantly changed, it is recommended that new outcome data be obtained to evaluate the effectiveness of the new format.

Residents commonly ask about the general lack of mental health resources and the difficulty establishing mental health care within the time frames suggested in the SAMP. As noted in the 2009 US Preventive Services Task Force recommendation statement, depression screening should only be undertaken when appropriate "systems are in place for diagnosis, treatment and follow-up" 6 (p. 1224); nonetheless, even when appropriate care is available, having patients seen by a mental health professional within a short time frame can be daunting. Possible workarounds that have been identified are to have the patient seen by the primary care physician or assign a staff member to call the patient for regular telephone check-ins while the patient is waiting to see a mental health professional.

Effectiveness measures include only resident self-perception of knowledge and comfort in screening and managing adolescent depression and suicidal ideation. While the existing data are highly significant, the effectiveness data would be strengthened by obtaining objective measures of change in physician behaviors as well as patient outcome data. This is planned for future study.

Angela Stanley, PsyD: Assistant Professor, Division of Adolescent Medicine, Medical College of Wisconsin

Gayathri Chelvakumar, MD: Assistant Professor, Division of Adolescent Medicine, The Ohio State University College of Medicine

Paula Cody, MD: Assistant Professor, Division of Adolescent Medicine, University of Wisconsin School of Medicine and Public Health

Mandakini Sadhir, MD: Assistant Professor, Division of Adolescent Medicine, University of Kentucky College of Medicine

Melodee Nugent: Biostatistician, Medical College of Wisconsin

Raymond Hoffmann, PhD: Professor of Pediatrics, Medical College of Wisconsin; Associate Director, Division of Quantitative Health Sciences, Medical College of Wisconsin

Pippa Simpson, PhD: Professor of Pediatrics, Medical College of Wisconsin; Director, Division of Quantitative Health Sciences, Medical College of Wisconsin

\section{Disclosures}

None to report.

Funding/Support

None to report.

Prior Presentations

A related abstract was accepted and presented at the Society of Adolescent Health and Medicine 2015 Annual Meeting and the Pediatric Academic Societies 2015 Annual Meeting (instructional materials not included).

Ethical Approval

This publication contains data obtained from human subjects and received ethical approval.

\section{References}

1. Lewinsohn PM, Rodhe P, Seeley JR. Major depressive disorder in older adolescents: prevalence, risk factors, and clinical implications. Clin Psychol Rev. 1998;18(7):765-794. http://dx.doi.org/10.1016/S0272-7358(98)00010-5

2. Welcome to WISQARS. Centers for Disease Control and Prevention Web site. http://www.cdc.gov/injury/wisqars/. Updated December 8, 2015

3. Elster AB, Kuznets NJ. AMA Guidelines for Adolescent Preventive Services (GAPS): Recommendations and Rationale Baltimore, MD: Williams \& Wilkins; 1994.

4. Committee on Practice and Ambulatory Medicine, Bright Futures Periodicity Schedule Workgroup. 2014 recommendations for pediatric preventive health care. Pediatrics. 2014;133(3):568-570. http://dx.doi.org/10.1542/peds.2013-4096

5. Rosen DS, Elster A, Hedberg V, Paperny D. Clinical preventive services for adolescents: position paper of the Society for Adolescent Medicine. J Adolesc Health. 1997;21(3):203-214. http://dx.doi.org/10.1016/S1054-139X(97)00116-X 
6. US Preventive Services Task Force. Screening and treatment for major depressive disorder in children and adolescents: US Preventive Services Task Force recommendation statement. Pediatrics. 2009;123(4):1223-1228. http://dx.doi.org/10.1542/peds.2008-2381

7. Olson AL, Kelleher KJ, Kemper KJ, Zuckerman BS, Hammond CS, Dietrich AJ. Primary care pediatricians' roles and perceived responsibilities in the identification and management of depression in children and adolescents. Ambul Pediatr. 2001;1(2):91-98. http://dx.doi.org/10.1367/1539-4409(2001)001<0091:PCPRAP>2.0.CO;2

8. Parchman ML. Physicians' recognition of depression. Fam Pract Res J. 1992;12(4):431-43

9. Zuckerbrot RA, Jensen PS. Improving recognition of adolescent depression in primary care. Arch Pediatr Adolesc Med. 2006;160(7):694-704. http://dx.doi.org/10.1001/archpedi.160.7.694

10. Green C, Hampton E, Ward MJ, Shao H, Bostwick S. The current and ideal state of mental health training: pediatric program director perspectives. Acad Pediatr. 2014;14(5):526-532. http://dx.doi.org/1016/j.acap.2014.05.011

11. Biddle V. Online Adolescent Suicide Risk Assessment. MedEdPORTAL Publications; 2013. Available from: https://www.mededportal.org/publication/9352http://dx.doi.org/10.15766/mep_2374-8265.9352

12. Goldenberg M, Penaskovic K. Suicide Risk Assessment Team-Based Learning (TBL) Module. MedEdPORTAL Publications; 20 Available from: https://www.mededportal.org/publication/9088http://dx.doi.org/10.15766/mep_2374-8265.9088

13. Richardson LP, McCauley E, Grossman DC, et al. Evaluation of the Patient Health Questionnaire-9 Item for detecting major depression among adolescents. Pediatrics. 2010;126(6):1117-1123. http://dx.doi.org/10.1542/peds.2010-0852

Received: August 27, 2015 | Accepted: January 14, 2016 | Published: March 21, 2016 